\title{
Multiscale modeling of defect formation during solid-phase epitaxy regrowth of silicon
}

\author{
M. Prieto-Depedro, I. Romero, I. Martin-Bragado
}

\begin{abstract}
This work presents a multiscale approach to understanding the defect formation during the evolution of solid-phase epitaxy regrowth in Si. A molecular dynamics (MD) simulation technique has been used to elucidate the defect formation mechanisms, as well as to determine their nature. A hybrid lattice kinetic Monte Carlo (LKMC)-finite element method (FEM) model fed by the outcome of MD was subsequently implemented. It scales up the simulation times and sizes, while reproducing the important features of the defected regrowth predicted previously. FEM calculations provide the strain pattern due to the density variation between the amorphous and crystalline phases, which is then taken into account by the LKMC model by including the effect of the strain in the rates of recrystallization. Overall, this multiscale modeling provides a physical explanation of the generation of defects and its relation with the presence of strain. The model also captures the character of formed defects. It distinguishes two types: twins formed at $\{111\}$ planes and dislocations produced by the collapse of the two recrystallization fronts. Simulation results are validated by comparing them with significant experiments reported in the literature.
\end{abstract}

(C) 2014 Acta Materialia Inc. Published by Elsevier Ltd. All rights reserved.

Keywords: Solid-phase epitaxial regrowth; Multiscale; Finite element method; Molecular dynamics; Lattice kinetic Monte Carlo

\section{Introduction}

The reduction of microelectronic device dimensions is imposed by the demanding requirements of semiconductor manufacturing. These dimensions have been reduced significantly over the past 40 years, and today are approaching the physical limits of miniaturization [1]. A s a consequence, new problems have arisen, such as the short channel effect. Promising technologies, such as FDSOI and non-planar trigate devices, appear to be alternatives to device scaling in terms of junction formation [2]. Solidphase epitaxy regrowth (SPER) is a widely used technique since it enables the formation of highly activated and abrupt junctions.
SPER is a very complex phenomenon. It is well known to have anisotropic behavior: the rates of recrystallization are strongly dependent on the orientation of the crystalline substrate, with velocity ratios of 1:10:20 for (111), (011) and (001), respectively [3]. In addition, under certain circumstances SPER recrystallization produces a trace of defects $[4,5]$. Finally, SPER is strongly dependent on stress [6], and dopant and impurity concentrations [7]. Such stresses can be externally generated, but there is always an intrinsic component produced by the $\sim 2 \%$ volume expansion of the $\alpha$-Si with respect to c-Si [8].

This study is focused on multidirectional SPER, particularly in the 2-D case, having two recrystallization fronts 
with different rates. Multidirectional SPER has also been shown to result in imperfect growth, which may impact the performance of electronic devices. Complex shapes of recrystallization fronts, incompatible with simple models, have been studied experimentally [9], showing the formation of downturned edges, as well as their evolution to small triangular edge pockets of $\alpha$-Si bounded by $\{111\}$ planes. Moreover, mask-edge defects appear when different recrystallization fronts meet, which have been reported to be composed of either shear-type or $60^{\circ}$ type perfect dislocations [10]. More recently, these have been determined to be Frank partial loops [11]. As a result, different type of defects have been shown to appear after complete recrystallization has been reached, though their origin remains unclear.

The present work is aimed at modeling the defected regrowth in Si to give a further insight into the defect formation mechanisms, and to shed light on their effect on the regrowth processes and their relation with the internal strains generated by the amorphous phase. Three different techniques, finite element method (FEM), molecular dynamics (MD) and lattice kinetic Monte Carlo (LKMC), are used in a multiscale approach to reach our goal. FEM calculations provide the strain pattern generated by the volume expansion of the $\alpha$-phase. The fundamental mechanisms of defect generation, as well as its character, are determined by MD simulations. Finally, physical conclusions obtained by MD are extended into the LKMC model. The LKMC method, integrated with FEM for the mechanical calculations, allows one to simulate realistic sample sizes and annealing temperatures. Finally, the results obtained via the techniques are validated by comparing them to significant experiments; in particular, the evolution of the recrystallization fronts is examined for two different orientations of the substrate from which the epitaxy is templated: $\langle 110\rangle$-aligned line shaped $\alpha$-Si in (001) Si and $\langle 100\rangle$-aligned line shaped $\alpha$-Si (011) Si.

\section{Finite element method}

This study begins with the calculation of the initial strain pattern due to density variation between the $\alpha$ - and the c-phases. This is the first step to reaching our final goal, to determine if the presence of strain inhibits the recrystallization rates, thus inducing the generation of defects. These patterns are the basis of further work. Since the volumetric strain due to $\alpha-\mathrm{Si}$ is known, a finite element model of the elastic response is employed to determine the complete elastic fields. In this model, each cell is represented by a standard isoparametric hexahedral element with an elastic constitutive law that includes an eigendeformation corresponding to the volumetric strain [12]. The energy minimization of the complete body, including its boundary conditions, yields the displacement field in the instantaneous equilibrium, and can be used to derived local gradients like the strains, stresses or other quantities of interest.
Fig. 1 shows the dependence of the sample size on the presence of shear strain computed with our simple FEM model. Fig. 1(a) and (b) corresponds, respectively, to an $\alpha$-Si rectangular region of $3.5 \times 13\left(\mathrm{~nm}^{2}\right)$ and $40 \times$ $143\left(\mathrm{~nm}^{2}\right)$ embedded in a c-Si template of $4 \times 14.0\left(\mathrm{~nm}^{2}\right)$ and $42 \times 146\left(\mathrm{~nm}^{2}\right)$. Strains are seen to appear in the near vicinity of the interfaces that accommodate the volumetric expansion of $\alpha$-Si. In the figure, (a) for very small amorphous regions of around $10 \mathrm{~nm}$, which can be simulated within a reasonable computational time using MD, shear strain is almost negligible. (b) For sizes an order of magnitude larger, which are realistic in devices, the contribution of shear strain is important. Consequently, if the effects of internally produced shear stress are to be studied using $\mathrm{MD}$, one has to emulate such structures by applying external compressions to the nanometric structures, similarly to the work done in Ref. [11].

\section{Molecular dynamics}

\subsection{Model and set up}

MD is a well-known technique based on the resolution of the Newton equations of motion of each constituent particle of the system, their positions followed in time being the outcome of the simulation [13]. This makes MD a suitable tool with which to simulate recrystallization processes. Classical interatomic potentials are used to describe the interactions between atoms. In this study, the Tersoff interatomic potential in its third parametrization (T3) is used [14]. T3 accurately reproduces the properties of both the $\alpha$ - and c-phases of Si [15], so it gives a reasonable description of the SPER from $\alpha-c$ interfaces [16]. This potential has a limitation, in that it predicts an excessive high melting temperature, $2400 \mathrm{~K}$, which is well above the experimental value, $1685 \mathrm{~K}$ [17]. However, this weakness is the key to the observation of SPER using MD, since the phenomenon is fast enough for the technique at $\sim 1500 \mathrm{~K}$ [18]. Moreover, $\mathrm{T} 3$ has been shown to be reliable for qualitatively modeling
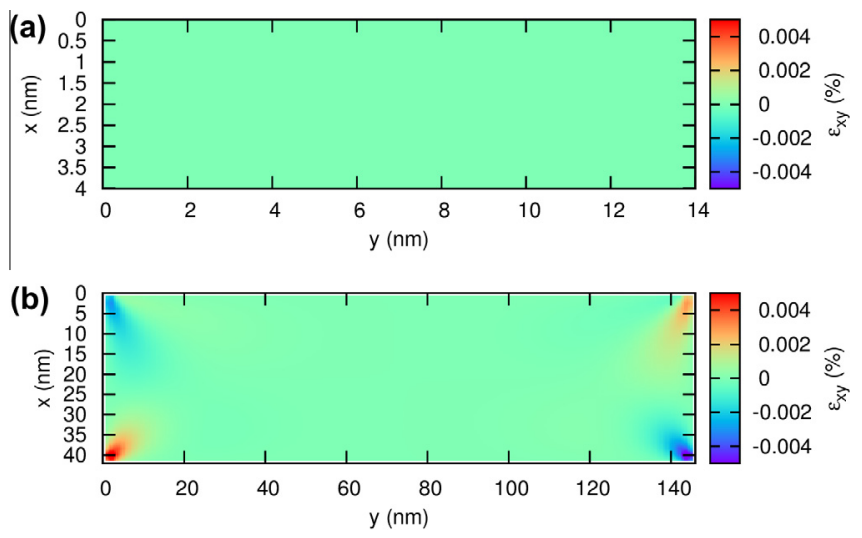

Fig. 1. Shear strain patterns generated by volume expansion of $\alpha$-Si in a box-shaped amorphized structure for a (a) MD domain size and (b) a LKMC domain size. 
the formation of defects during SPER in Si nanodevices, as reported by Marques et al. in Ref. [19] and Lampin and Krzeminski in Ref. [16].

To study the formation of defects in multidirectional SPER, the schematic structure in Fig. 2 is used. It consists of four different regions. A few atomic planes at the bottom are held fixed in their perfect lattice positions. These frozen atoms act as a substrate, identified as grey zones in the figure. c-Si atoms at lined regions serve as the seeds for recrystallization, being in direct contact with the $\alpha$-Si that is to be recrystallized (white area). The $\alpha$-Si region placed at the top of the box acts as a filled trench: atoms at this region are also fixed in their random positions.

In this work, the amorphous region is not obtained by simulating ion implantation, as the large number of implantations required and the subsequent relaxation of the box would have made the process infeasible. Instead, a faster method is used to create the computational structure: knowing the exact number $N$ of atoms in the c-Si region to be amorphized, these are added to the system at randomly generated coordinates followed by an energy minimization of the system using the conjugate gradient method. The MD cell is preannealed at $1000 \mathrm{~K}$ for $20 \mathrm{ps}$ and then annealed at $2000 \mathrm{~K}$ for $4 \mathrm{~ns}$.

Periodic boundary conditions are applied in parallel directions to the $\alpha-c$ interface, while in the normal direction atoms are sandwiched between the $\alpha$-top slab, simulating the isolation by $\mathrm{SiO}_{2}$ present in real samples, and the c-substrate at the bottom. Equations of motion are integrated using the Verlet algorithm with a time step of $0.002 \mathrm{fs}$. The regrowth of $\langle 110\rangle$-aligned box-shaped in $(001) \mathrm{Si}$ has been simulated using a $61 \times 148 \times 38\left(\AA^{3}\right)$ simulation cell, containing 19,553 atoms, and a $76 \times 150 \times 30\left(\AA^{3}\right)$ one with 18,144 atoms for the $\langle 100\rangle$-aligned box-shaped in (0.11) Si.

Based on the assumption that the anomalous recrystallization is due to the presence of a strain pattern, MD simulations are carried out under two different conditions: (a) in the absence of any external stress applied, referred to the pattern in Fig. 1(a) and (b) under an external uniaxial compressive stress applied to induce the internal strains that a bigger structure would feel, as previously explained. In the latter case, MD simulations are performed by compressing the simulation box by $0.5 \mathrm{~nm}$ on each side in the $y$ direction and maintaining the constraint during the whole annealing time. This compression induces an external stress within the range of 5-7 GPa, depending on the orientation of the crystalline substrate. From MD simulations, the threshold stress to form defects is confirmed to exceed $\sim 2 \mathrm{GPa}$.

\subsection{Results and discussion}

In the following, the results obtained using the LAMMPS [20] simulator are presented. Relaxed structures are annealed until their complete recrystallization is reached. The rectangular shape of the amorphous region allows one to observe the pinch-off point of the two growth fronts (not shown here). MD simulation results of the SPER process after $4 \mathrm{~ns}$ of annealing at $2000 \mathrm{~K}$ show the formation of twinning (see Fig. 3). However, the MD model does not catch the slower regrowth at the corner of the boxshaped $\alpha$-region, hence mask-edge defects are not formed. This results validates the idea that, in the absence of significant strain, only partial defective recrystallization is produced.

The next step to fully understand the defected SPER by MD simulations is to force the generation of defects by applying external stress. Figs. 4 and 5 present different stages of the annealing for the two orientations of the template under study. In both cases, SPER produces a characteristic $\{111\}$ faceting in the lateral fronts, while the recrystallization proceeds rectilinearly in the vertical direction, as shown in Figs. 4(a) and 5(a). In particular, the recrystallization of the $\langle 110\rangle /(001) \alpha$-Si shows the previously mentioned downturned edges see Fig. 4(b). In the case of $\langle 100\rangle /(011) \alpha-\mathrm{Si}$, the intermediate stage observed in Fig. 5(a) shows how the deviation of rectilinear shapes is even greater than in $\langle 110\rangle /(001)-\mathrm{Si}$. Lateral facet points are well defined due to the prominent angle between recrystallization fronts.

These characteristic recrystallization processes are explained according to the model proposed in Ref. [9]: assuming that $\{111\}$ microscopic facets are stable once formed, the $\alpha$-Si recrystallizes templated by those planes. Under this consideration, the two recrystallization fronts meeting point occurs at an angle of $70.6^{\circ}$ for $\langle 10\rangle /(001)$ $\mathrm{Si}$ and $109.4^{\circ}$ for $\langle 100\rangle /(011)-\mathrm{Si}$. The MD simulation results shown in Figs. 4 and 5 match the geometrical predictions well.

Once the recrystallization is completed, the defects formed are analyzed. Fig. 6 shows the final structures in the two orientations considered. The regrowth process produces twinning formation from the development of $\{111\}$
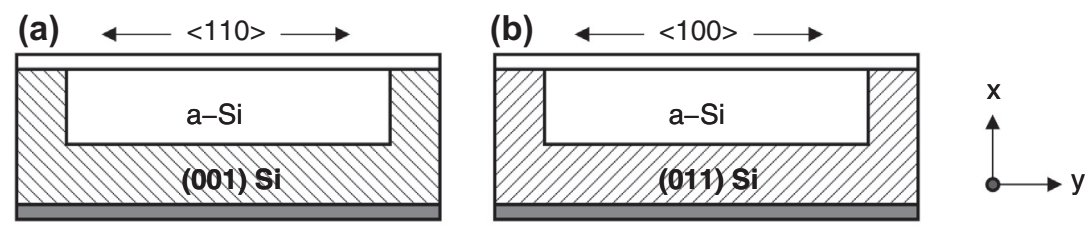

Fig. 2. Cross-sectional schematics of $\langle 110\rangle$-aligned shapes in (a) (001) Si and (b) (011) Si. Grey slabs represent fixed atoms used as substrate. Atoms in the top slab are identified with filled trenches. 
(a)
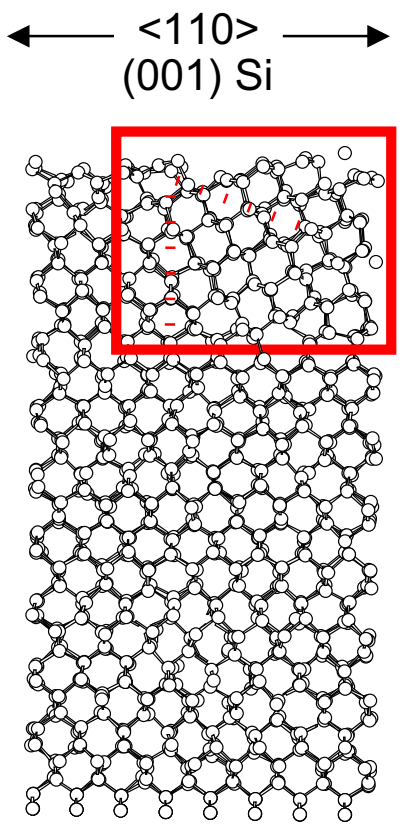

(b)
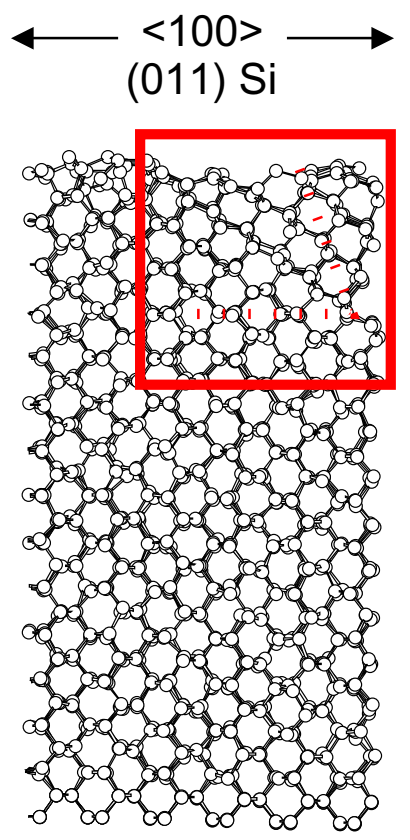

Fig. 3. Cross sectional MD free-stress simulation snapshots of $\langle 110\rangle$-aligned box-shaped $\alpha$-Si regions in (a) $\operatorname{Si}(001)$ and (b) $\operatorname{Si}(011)$ : after annealing at $2000 \mathrm{~K}$ for $4 \mathrm{~ns}$. Twinning formation is identified with red lines. (For interpretation of the references to color in this figure legend, the reader is referred to the web version of this article.)

(a)

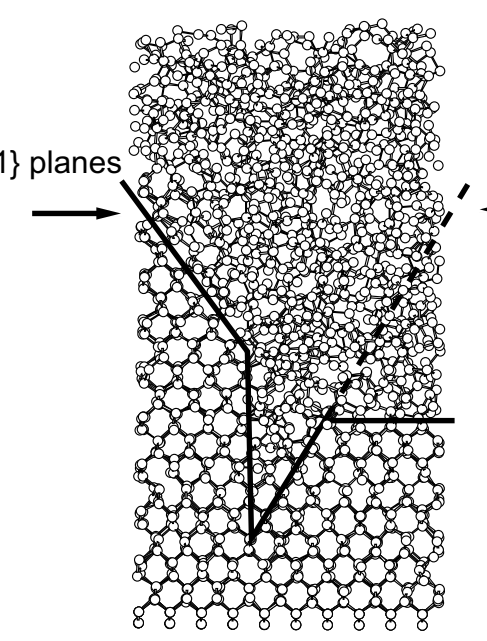

(b)

$\{111\}$ planes

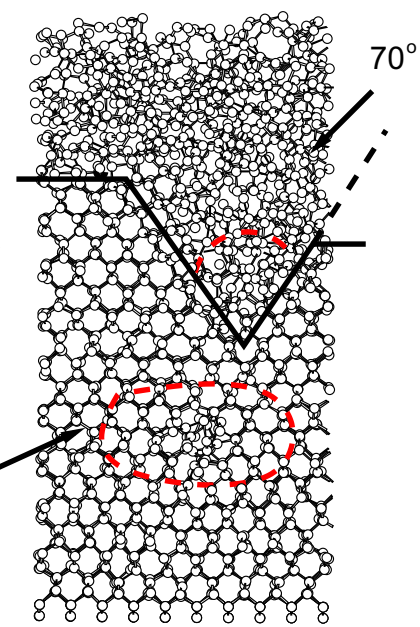

Fig. 4. Cross sectional MD simulation snapshots of $\langle 110\rangle$-aligned box-shaped $\alpha$-Si regions in $(001)$ Si under compressive stress: after annealing at $2000 \mathrm{~K}$ for (a) $0.3 \mathrm{~ns}$ and (b) $0.5 \mathrm{~ns}$. Dashed-red lines indicate defected regrowth zones. (For interpretation of the references to color in this figure legend, the reader is referred to the web version of this article.)

planes, identified in the red zones. A twin is defined as a $60^{\circ}$ rotation with respect to one bond in the original tetrahedron. The rotation angles obtained from the simulation results are about $65^{\circ}$ for both orientations considered. Dislocations appears at the pinch-off point of the fronts. Shen et al. [11] confirmed the defects to be Frank partial loops with Burgers vector $a_{0} / 3\langle 11\rangle$, by contrasting their MD simulation results with experimental TEM images.

In contrast to the recrystallization of the sample under no applied external strain, when compressing the box, SPER is significantly slowed down at the corners, as clearly seen in Figs. 4(a) and 5(a). This induces the formation of $\{111\}$ facets which, after collapsing, leave a pattern of mask-edge defects. These linked events are clearly related to the presence of shear strain in the vicinity of the $\alpha-c$ interface, as also stated in Ref. [21]. MD goes further by distinguishing the different natures of the formed defects. Two different, non-equivalent types of defect are produced during SPER: microtwins, which are developed due to the stability of the $\{111\}$ planes, and dislocations, which are formed when two fronts, originally from the same substrate, produced by the slow-down of shear strain, collapse. 

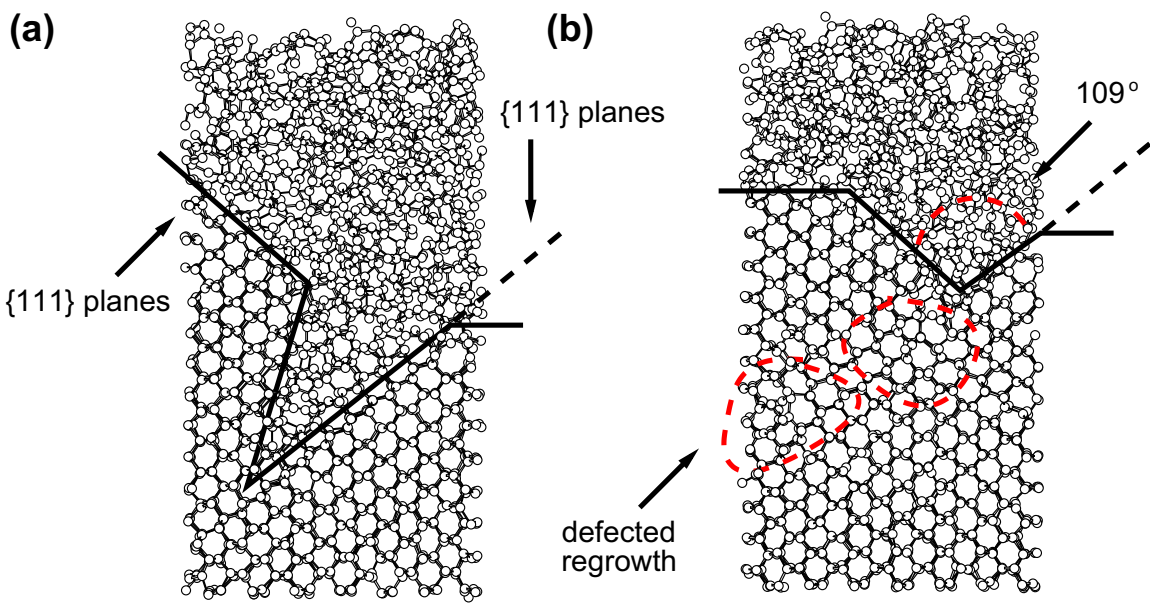

Fig. 5. Cross sectional MD simulation snapshots of $\langle 100\rangle$-aligned box-shaped $\alpha$-Si regions in $(011)$ Si under compressive stress: after annealing at $2000 \mathrm{~K}$ for (a) $0.6 \mathrm{~ns}$ and (b) $1.2 \mathrm{~ns}$. Dashed-red lines indicate defected regrowth zones. (For interpretation of the references to color in this figure legend, the reader is referred to the web version of this article.)
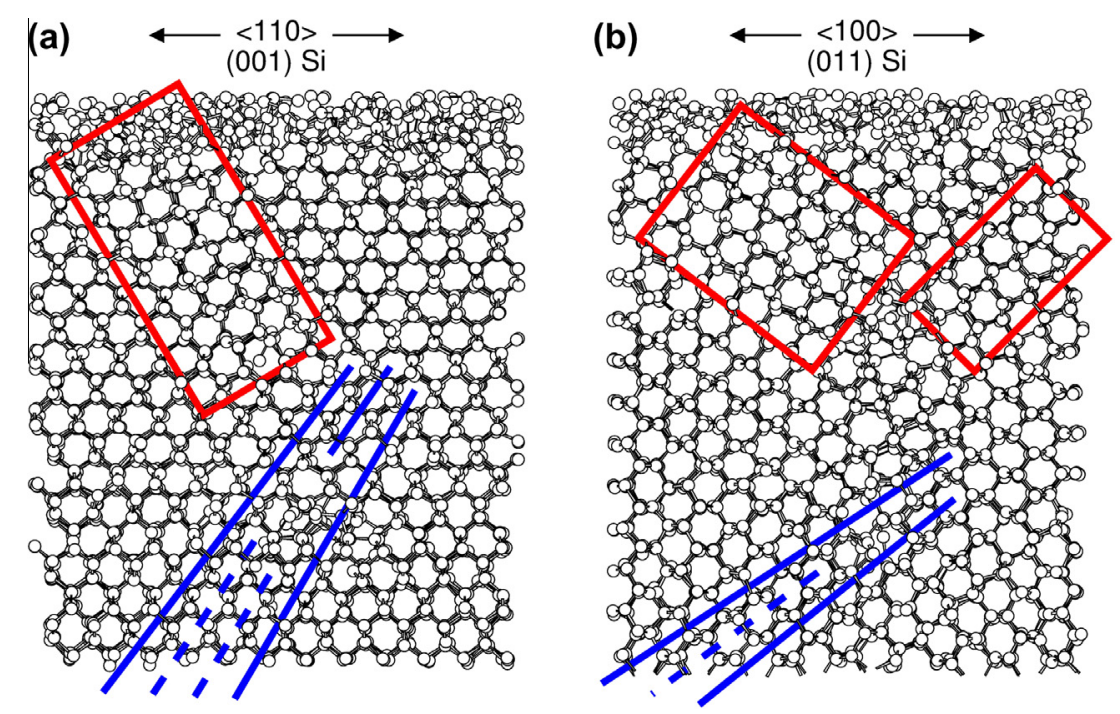

Fig. 6. Cross sectional images of the complete regrowth of (a) $\langle 110\rangle$-aligned box-shaped $\alpha$-Si regions in (001)-Si and (b) $\langle 100\rangle$-aligned box-shaped $\alpha$-Si regions ( $\left.\begin{array}{lll}0 & 1 & 1\end{array}\right)-\mathrm{Si}$, under compressive external stress. Blue lines identify the formed dislocations. Red lines indicate the presence of twins. (For interpretation of the references to color in this figure legend, the reader is referred to the web version of this article.)

\section{Lattice kinetic Monte Carlo}

\subsection{Model description}

An atomistic LKMC method to study the process of evolution and defect formation during SPER is used. The model, based on the ideas of Drosd and Washburn [3], has been implemented in the modular Monte Carlo simulator MMonCa [22,23] according to Refs. [24,25]. The model describes the amorphous to crystalline transitions by considering the rates of recrystallization events as the probability per unit of time of an atom to join the c-phase. These probabilities depend on the neighborhood of the considered configuration, which in turn is determined by the local regrowth plane. There are three different configurations, $\{100\},\{011\}$ and $\{111\}$, requiring 1,2 and 3 atoms, respectively, to join the c-phase (see Fig. 1 in Ref. [26]). The stress-free recrystallization frequency within the transition state theory is given by:

$K$ (site) being the site-dependent prefactor and $E_{\text {activation }}=2.7 \mathrm{eV}$ the activation energy for $\mathrm{Si}$, with $k_{B} T$ having the usual meaning.

The MD simulations previously shown indicate that there are three important physical mechanisms that need to be scaled up into LKMC: (i) the formation of twins; (ii) the slowed recrystallization near the corners as a consequence of the shear strain; and (iii) the collapse of the two recrystallization fronts induced by (ii) and further formation of dislocations. The formation of twins is included in the model by using the technique previously explained in Ref. [27]. The strain is included in the calculations by coupling the LKMC model to the FEM module explained 
above. The feedback between both techniques is defined. Iteratively, the LKMC transforms $\alpha$-Si into c-Si. The structure is accounted for by the FEM module, which computes the strains associated with the volume expansion of $\alpha$-Si and transfers the strains back to modify the regrowth rates. Finally, the collapse of the two fronts is a natural cause of the previous steps and does not need to be explicitly modeled. The correction to the activation energy is introduced in two steps. First, the non-shear corrections are introduced as explained in Ref. [28], then an extra slow-down produced by the shear strain $\epsilon_{x y}$, in agreement with the experimental observations of Ref. [21], is accounted for as:

$E=E_{\text {activation }}+\epsilon_{x y}^{2} * K$

$K=9 \times 10^{3} \mathrm{eV}$ being a parameter that has been fitted to properly reproduce experimental observations.

\subsection{Results and discussion}

In this section, the results obtained by the LKMC model, limited to the $\langle 110\rangle$-aligned amorphous regions in $\mathrm{Si}(001)$ and the $\langle 100\rangle$-aligned $\alpha$-Si in (011) Si, are analyzed. Simulation boxes of $42 \times 146 \times 20\left(\mathrm{~nm}^{3}\right)$ were run at temperatures of $550^{\circ} \mathrm{C}$ for a maximum time of $15,000 \mathrm{~s}$. Here, no external stresses were applied: the size of the amorphous region was big enough to produce internal strains that impacted on the recrystallization significantly.

Fig. 7 shows several snapshots at different stages of the SPER process for two orientations of the Si substrate. For clarity, only the atoms at the a-c interfaces are represented. Fig. 7(b) and (g) shows the formation of $\{111\}$ facets, which acts as a template for the progressive recrystallization. The regrowth at the corners is seen to be slowed. The meeting point of the fronts occurs at an angle of $74^{\circ}$ for (001) (see Fig. 7(d) for (011) (see Fig. 7(h)), in good agreement with the predicted values from the geometrical approach in Ref. [9] (previously mentioned in Section 3.2). In particular, for $\mathrm{Si}(001)$, as observed before in $\mathrm{MD}$ simulations, when the vertical regrowth front reaches the $\{111\}$ planes developed at the lateral fronts, the expected formation of downturned edges occurs, which evolve into edge triangular pockets as shown in Fig. 7(e). These pockets remain after complete regrowth has been achieved. For $\operatorname{Si}(011)$, the lateral facet points are well defined due to the prominent angle faces of the two fronts, shown in Fig. 7(h). In general, the agreement with the experimentally observed evolution of similar topologies shown in Refs. [29-31] is remarkable, and shows how the successful transfer of meaningful information between $\mathrm{MD}$ and LKMC, and the hybridization of LKMC with FEM, provides a reliable technique for simulating realistic experiments.

Finally, Fig. 8 provides a validation of the extended defects predicted by LKMC by comparison with the cross-sectional transmission electron microscopy (XTEM) images reported by Saenger et al. [9]. There is good agreement between them, although some discrepancies are also observed. It is interesting to note that the formation of twins predicted by LKMC, as well as by MD, is not observed in the XTEM images. Nevertheless, we can explain these differences by noting that, in contrast to the original model of Saenger et al.[9], our models predict two types of defect - twining and dislocation formation - (a)

(b)

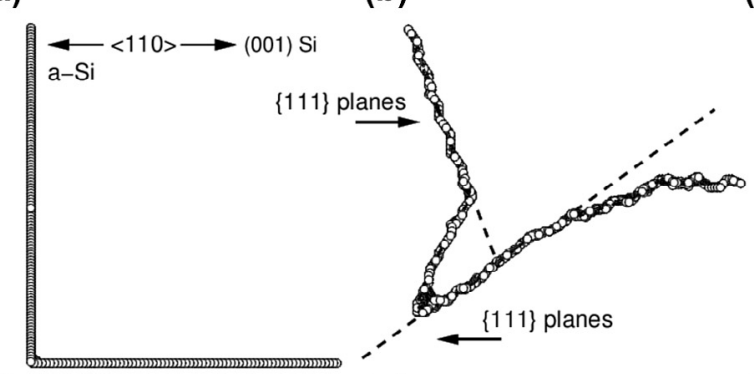

(f)

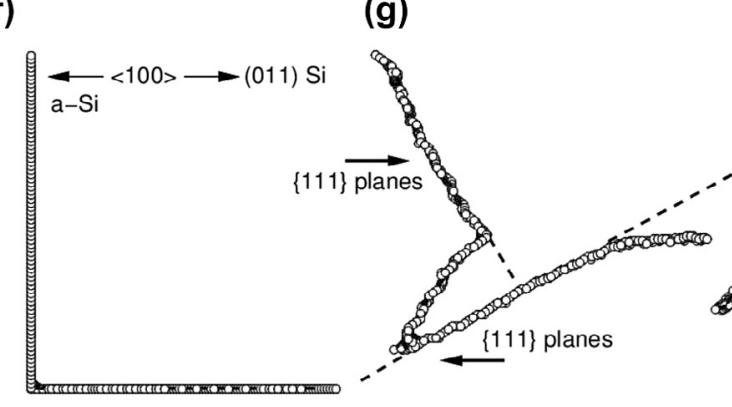

(c)

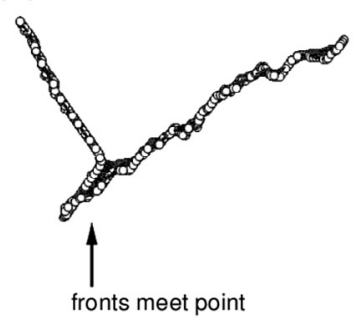

(d)

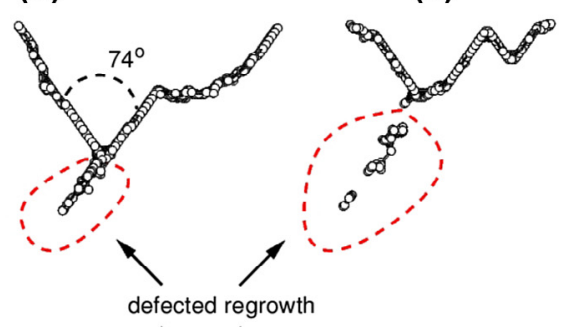

(e) (h)

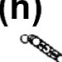

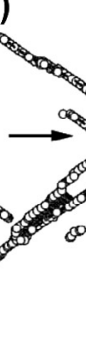
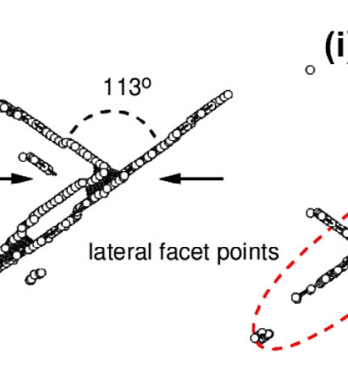
(i) 


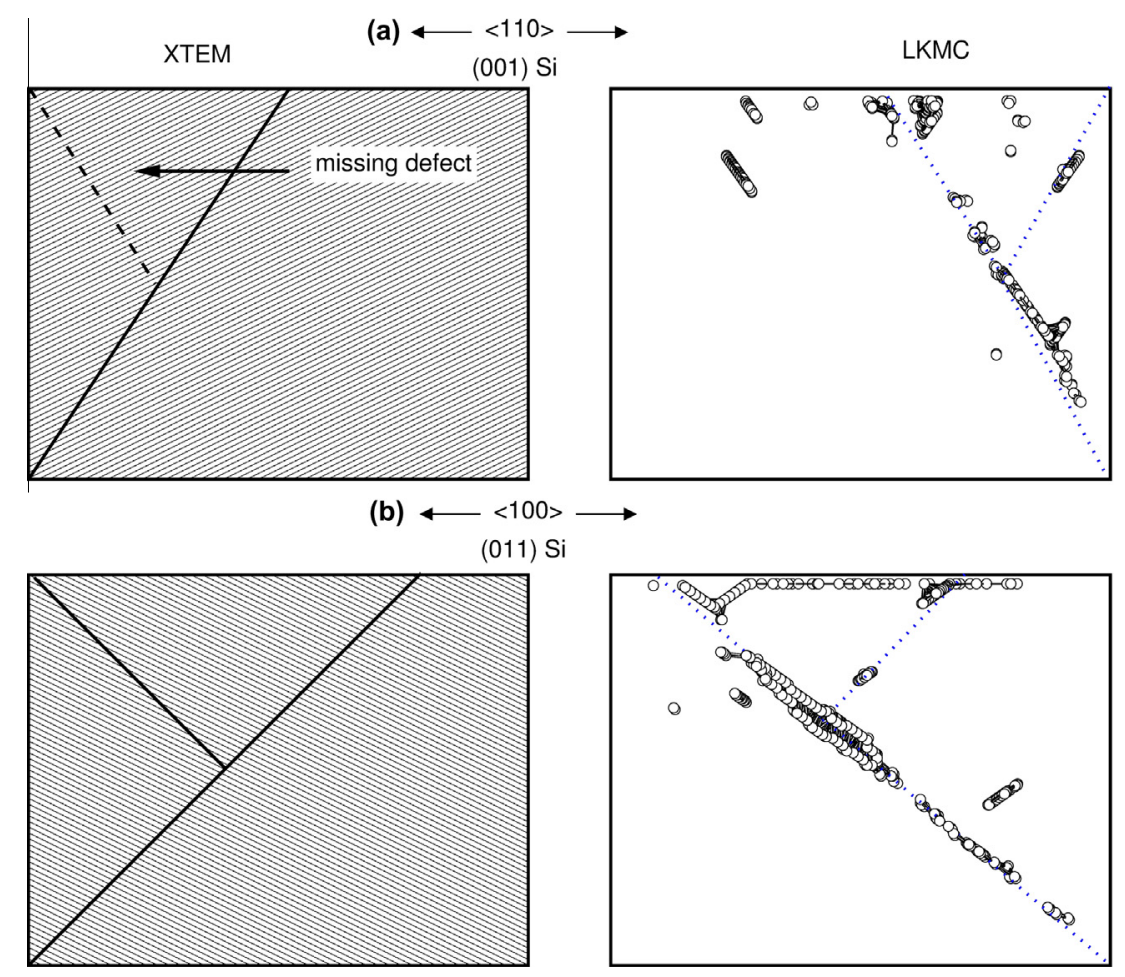

Fig. 8. Comparison of formed defects observed by XTEM reported in Ref. [9] (left) and predicted by the LKMC model (right) for (a) $\langle 110\rangle$-aligned boxshaped $\alpha$-Si regions in (00 1)-Si and (b) $\langle 100\rangle /\left(\begin{array}{lll}0 & 1 & 1\end{array}\right)$-Si.

and that only the dislocations seem to be clearly visible in the microscopy images without precluding that twins are also present in the samples in the positions predicted by our models. According to the XTEM images in Ref. [9], the lattice resolution does not allow the detailed observation of defects smaller than $2 \mathrm{~nm}$ in length. Otherwise, the invisibility can be explained by the viewing direction during TEM examination not being adequate to observe twins formed at $\{111\}$ planes.

\section{Conclusions}

A comprehensive multiscale description of defect formation during SPER is presented. First, MD simulations have provided a physical explanation for the SPER microscopic mechanisms of the generation of defects, and allow us to determine their character. The simulation results show two types of defect. The formation of twins is induced by the stability of $\{111\}$ facets, which, once formed, template the further recrystallization. The second type are dislocations, the formation mechanism of which has been determined by checking the existing relation between them and the presence of strain due to the volume expansion of the $\alpha$-Si. MD simulations show that there is no dislocation generation in the absence of strains, but that, when significant strains are achieved in the samples (either by applying external forces, as in our small MD domains, or by the volumetric expansion on $\alpha$-Si in microscopic samples), a slow-down in the regions with high shear strain produces the evolution of two ledges that collapse and evolve into Frank partial loops. However, due to the timeand size-scale limitations of classical MD, the simulation conditions are dissimilar to those in actual devices. Consequently, to extend the study into real applications, an LKMC model fed with the information obtained by MD is considered. The model hybridizes with an FEM module to obtain the strain pattern induced by the $\alpha$-Si. It introduces an extra term in addition to the activation energy, which decreases the frequency of recrystallization when shear strain is present. As a result, the regrowth process is inhibited, inducing the generation of defects. The extensive defects predicted by LKMC matched those observed in XTEM images well, confirming that the recrystallization templated by $\{111\}$ planes is the main cause for twinning formation, while the origin of dislocation nucleation at the pinch-off points is attributed to the slower regrowth at the corners induced by the strain.

\section{Acknowledgements}

The authors acknowledge partial funding by Abengoa Research. I.M.B. also acknowledges partial funding from the "Subprograma Ramón y Cajal" fellowship RYC2012-10639 by the Spanish Ministry of Economy and Competitiveness and partial funding of the project MASTIC (PCIG09-GA-2011-293783) by Marie Curie Actions Grant. The authors acknowledge B. Sklenard for his critical reading of the manuscript. 


\section{References}

[1] Thompson S, Parthasarathy S. Mater. Today 2006;9:20.

[2] A. Medury, K. Mercha, R. Ritzenthaler, A. De Keersgieter, T. Chiarella and N. Collaert, N. Bhat, 13th International Conference on Ultimate Integration on Silicon (ULIS), 2012, p. 113.

[3] Drosd R, Washburn J. J. Appl. Phys. 1982;53:397.

[4] Burbure N, Rudawski N, Jones K. Electrochem. Solid State 2007; 10:H184.

[5] Saenger K, de Souza J, Fogel K, Ott J, Sung C, Sadana D, et al. J. Appl. Phys. 2007:101.

[6] Rudawski N, Jones K. Scr. Mater. 2009;61:327.

[7] Olson G, Roth J. Mater. Sci. Rep. 1998;3:1.

[8] Custer J, Thompson M, Jacobson D, Poate J, Roorda S, Sinke W, et al. Appl. Phys. Lett. 1994;64:437.

[9] Saenger K, Fogel K, Ott J, Sadana D. J. Appl. Phys. 2007:101.

[10] Rudawski N, Jones K, Morarka S, Law M, Elliman R. J. Appl. Phys. 2009:105.

[11] T. Shen, Y. Tung, Y. Cheng, D. Chiou, C. Chen, C. Wu, Y.M. Sheu, H. Tsai, C. Huang, G. Hsieh, G. Tsai, S. Fung, J. Wu, C. Diaz, in: Electron Devices Meeting, IEEE International, 2012, pp. 30.1.130.1.4.

[12] Hughes T. The Finite Element Method. New Jersey, Englewood Cliffs: Prentice-Hall Inc.; 1987.

[13] Frenkel D, Smit B. Understanding Molecular Simulation. From Algorithms to Applications. Academic Press; 2002.
[14] Tersoff J. Phys. Rev. B 1989:39.

[15] Cook S, Clancy P. Phys. Rev. B 1993:47.

[16] Lampin E, Krzeminski C. J. Appl. Phys. 2009:106.

[17] Mayer J, Lau S. Electronics Materials Science for Integrated Circuits in Si and GaAs. MacMillan; 1990.

[18] Lampin E, Krzeminski C. J. Appl. Phys. 2011:109.

[19] Marqués L, Pelaz L, Santos I, López P, Duffy R. J. Appl. Phys. 2012:111.

[20] $<$ http//lammps.sandia.gov $>$.

[21] Shin Y, Lee J, Park M, Kang H. J. Cryst. Growth 2001;231:107.

[22] Martin-Bragado I, Rivera A, Valles G, Gomez-Selles J, Caturla M. Comput. Phys. Commun. 2013;184:2703.

[23] $<$ http://www.materials.imdea.org/MMonCa $>$.

[24] Martin-Bragado I, Moroz V. Appl. Phys. Lett. 2009:95.

[25] Martin-Bragado I, Moroz V. Appl. Phys. Lett. 2011:98.

[26] Martin-Bragado I. Scr. Mater. 2012;66:186.

[27] Martin-Bragado I, Sklenard B. J. Appl. Phys. 2012:112.

[28] Sklenard B, Batude P, Rivallin P, Tavernier C, Cristoloveanu S, Martin-Bragado I. Appl. Phys. Lett. 2013;102:151907.

[29] Morarka S, Rudawski N, Law M, Jones K, Elliman R. J. Appl. Phys. 2009:105.

[30] Morarka S, Rudawski N, Law M. J. Vacuum Sci. Technol. B 2008:26:357

[31] Cerva H, Küsters K. J. Appl. Phys. 1989;66:4723. 